\title{
COMMENT
}

\section{Which is better, HLA-matched sibling or haploidentical transplantation?}

\author{
Xiaodong Zheng ${ }^{1,2}$ and Zhigang Tian ${ }^{1,2}$ \\ Cellular \& Molecular Immunology (2021) 18:1347; https://doi.org/10.1038/s41423-021-00640-9
}

Allogeneic hematopoietic stem cell transplantation (allo-HSCT) is a curative treatment for patients with acute myeloid leukemia (AML). ${ }^{1}$ HLA-matched sibling donors (MSDs) remain the best donor source because of rapid hematopoietic and immunologic reconstitution and lower incidences of infections and acute graftversus-host disease (GVHD), but only $25-30 \%$ of patients can undergo HLA-matched sibling donor transplantation (MSDT). For most patients, alternative donor sources, such as HLA-matched unrelated donors, HLA-haploidentical donors or umbilical cord blood, may be better options. ${ }^{2}$

Haploidentical hematopoietic stem cell transplantation (haploHSCT), which is available for nearly all patients, initially generated poor outcomes due to high rates of graft failure and GVHD. However, in recent decades, better prevention of GVHD and optimization of transplantation techniques, including the use of T-cell depletion allografts, ${ }^{3,4}$ posttransplant cyclophosphamide (PTCY), ${ }^{5}$ G-CSF-mobilized allografts and antithymocyte globulin, have led to marked improvements in outcomes for haplo-HSCT. ${ }^{4,7}$ Considering HLA mismatches between haploidentical donors and recipients, NK cells or T cells in patients receiving haplo-HSCT should exhibit stronger graft-versus-leukemia (GVL) effects. ${ }^{8}$ However, to ensure higher rates of engraftment and decrease the incidence of GVHD, more intensive immunosuppression is administered after haplo-HSCT. ${ }^{9}$ It is unclear whether the immunosuppressive environment could reduce GVL effects in patients undergoing haplo-HSCT.

In the present study, Guo et al. developed nonirradiated leukemia mouse models that carried the human AML-ETO or MLLAF9 gene. ${ }^{10}$ They found that haplo-HSCT treatment could significantly extend survival and reduce tumor burden in AMLETO or MLL-AF9 leukemia mice. Increasing numbers and effector functions of T cells and NK cells in haplo-HSCT-treated mice might contribute to enhanced GVL effects. The data from the clinical trial, which included $135 \mathrm{AML}$ patients who received haplo-HSCT or MDST treatment, were consistent with those from AML mouse models. Haplo-HSCT carries a lower leukemia burden, a lower risk of relapse, better leukemia-free survival, and better overall survival but a significantly increased risk of acute grade II-IV GVHD. Interestingly, one year after transplantation, $\mathrm{T}$ cells from the patients undergoing haplo-HSCT still presented higher cytotoxic activity against leukemia cells than did those from the patients undergoing MSDT. Moreover, the group's previous study showed higher expression of CD107a on NK cells in haplo-HSCT-treated patients. ${ }^{11}$ These results indicated that GVL effects were enhanced in haplo-HSCT-treated AML patients. This provided one possible explanation for why AML patients receive more favorable clinical benefits from haplo-HSCT.

In summary, haplo-HSCT has multiple advantages, such as widespread availability of stem cell sources, enhanced GVL effects, improved immunologic reconstitution, and favorable clinical benefits. It is foreseeable that HLA matching will no longer be the top priority in donor selection for AML patients in the near future.

\section{ADDITIONAL INFORMATION}

Competing interests: The authors declare no competing interests.

\section{REFERENCES}

1. Blume, K. G. et al. Bone-marrow ablation and allogeneic marrow transplantation in acute leukemia. N. Engl. J. Med. 302, 1041-1046 (1980).

2. Kanakry, C. G., de Lima, M. J. \& Luznik, L. Alternative donor allogeneic hematopoietic cell transplantation for acute myeloid leukemia. Semin. Hematol. 52, 232-242 (2015).

3. Aversa, F. et al. Successful engraftment of T-cell-depleted haploidentical "threeloci" incompatible transplants in leukemia patients by addition of recombinant human granulocyte colony-stimulating factor-mobilized peripheral blood progenitor cells to bone marrow inoculum. Blood 84, 3948-3955 (1994).

4. Aversa, F. et al. Full haplotype-mismatched hematopoietic stem-cell transplantation: a phase II study in patients with acute leukemia at high risk of relapse. J. Clin. Oncol. 23, 3447-3454 (2005).

5. Luznik, L. et al. HLA-haploidentical bone marrow transplantation for hematologic malignancies using nonmyeloablative conditioning and highdose, posttransplantation cyclophosphamide. Biol. Blood Marrow Transplant. 14, 641-650 (2008).

6. Lu, D. P. et al. Conditioning including antithymocyte globulin followed by unmanipulated HLA-mismatched/haploidentical blood and marrow transplantation can achieve comparable outcomes with HLA-identical sibling transplantation. Blood 107, 3065-3073 (2006).

7. Wang, Y. et al. Haploidentical vs. identical-sibling transplant for AML in remission: a multicenter, prospective study. Blood 125, 3956-3962 (2015).

8. Kolb, H. J., Schmid, C., Barrett, A. J. \& Schendel, D. J. Graft-versus-leukemia reactions in allogeneic chimeras. Blood 103, 767-776 (2004).

9. Sidlik-Muskatel, R. \& Reisner, Y. Toward safer haploidnetical hematopoietic stem cell transplantation. Bone Marrow Transplant. 54, 733-737 (2019).

10. Guo, $\mathrm{H}$. et al. Dynamic immune profifiling identififies the stronger graft-versusleukemia (GVL) effects with haploidentical allografts compared to HLA-matched stem cell transplantation. Cell. Mol. Immunol. 18, 1172-1185 (2021).

11. $\mathrm{Hu}, \mathrm{L}$. J. et al. NK cell reconstitution following unmanipulated HLA-mismatched/ haploidentical transplantation compared with matched sibling transplantation. Sci. China Life Sci. 63, 781-784 (2020).

\footnotetext{
'Division of Molecular Medicine, Hefei National Laboratory for Physical Sciences at Microscale, the CAS Key Laboratory of Innate Immunity and Chronic Disease, School of Life Sciences, University of Science and Technology of China, Hefei, China and ${ }^{2}$ Institue of Immunology, University of Science and Technology of China, Hefei, China Correspondence: Xiaodong Zheng (zhxdd@ustc.edu.cn)
}

Received: 7 January 2021 Accepted: 9 January 2021

Published online: 20 April 2021 